\title{
SUBJECT MATTER IN BRIEF
}

\author{
British Journal of Nutrition, Vol. 57, No. 1, January 1987
}

\section{Papers relevant to CLINICAL AND HUMAN NUTRITION}

Growth of artificially reared rats. The technique of artificially rearing rats on milk substitutes is potentially extremely useful for developmental studies, but it is often attended by high morbidity and poor growth. Starting artificial rearing on expressed rats' milk and then changing over either gradually or abruptly to milk substitute was much more successful.

25-Hydroxy vitamin $D$ in human saliva. Vitamin D can be measured in saliva, the amount being $1.2 \%$ of that present in serum. The values obtained vary throughout the day and may relate to dietary intake and subject's ethnic origin. Salivary vitamin D variations may have a physiological role in the mineralization of the developing tooth.

\section{Papers on GENERAL NUTRITION}

Trace minerals, casein and cholesterolaemia. Casein diets induce hypercholesterolaemia in rabbits but soya-bean protein diets do not. Little is known of the mechanism. This study in rabbits showed that when the zinc content of a casein diet was increased to equal that of a soya-bean diet, the increase in plasma cholesterol was negated.

Effects of dietary zine on $\mathbf{Z n}$ absorption in young rats. In rats maintained on diets of differing $\mathrm{Zn}$ contents, homeostatic control of $\mathrm{Zn}$ status was regulated by changes in both absorption and excretion when dietary $\mathrm{Zn}$ was low, and excretion alone at high $\mathrm{Zn}$ intakes. A role for intestinal metallothionein in either absorption or excretion was not established.

Intestinal transport of zinc. A basic study using piglet intestinal cell membranes showed two mechanisms for $\mathrm{Zn}$ absorption. One was passive and inhibited by other trace metals. The second was a carrier-mediated process specific to $\mathrm{Zn}$ (and cadmium) and slightly stimulated by low concentrations of the milk protein lactoferrin.

Control of rumen degradation of fresh forages. Dietary treatments to control excessive rumen degradation of forage protein were examined and marked responses to formaldehyde application were observed in rumen ammonia concentrations, and duodenal flows of non- $\mathrm{NH}_{3}$ nitrogen and microbial $\mathrm{N}$. In contrast, monensin had no effect on $\mathrm{N}$ metabolism, but increased propionate : acetate ratio. 
Wheat bran components and volatile fatty acids in rats. Giving wheat aleurone layers led to much higher volatile fatty acid concentrations in caecal digesta and hepatic portal venous plasma than giving the parent bran or its pericarp-seed coat fraction. This reflects the high content of fermentable polysaccharides in aleurone walls and their greater accessibility for the caecal microflora.

Nitrogen repletion by $\mathbf{N}$-depleted sheep. Lambs wholly nourished by intragastric infusion were depleted of $\mathrm{N}$ then repleted. Judged by $\mathrm{N}$ losses there was some evidence of immediate adaptation but no further long-term adaptation during depletion. Repletion was rapid (within a few days) but enhanced $\mathrm{N}$ accretion (compensatory growth) continued for weeks.

Rumen microbial protein synthesis. Continuous intraruminal infusion of soluble sugars markedly enhanced the synthesis of microbial protein in the rumens of cattle fed on grass silage. The absence of a rapidly fermentable carbohydrate source may therefore be an important factor underlying the poor utilization of silage nitrogen for rumen microbial protein synthesis.

Growth promoters and protein metabolism in lambs. The total protein in the longissimus dorsi and vastus lateralis muscles but not the vastus intermedius was increased by dietary clenbuterol ( $\beta$-2-adrenergic agonist). Circumstantial evidence suggests this was due to reduced protein degradation. The effects of a steroid implant on muscle were much less marked.

99-107

Net nitrogen absorption by beef heifers. Casein, soya-bean meal, maize-gluten meal or blood meal provided about two-thirds of $\mathrm{N}$ in four diets given to beef heifers. Although in vitro degradability of $\mathrm{N}$ varied about fourfold among diets, net absorption of ammonia- $\mathrm{N}$ and $\alpha$-amino- $\mathrm{N}$ was not affected markedly.

Effects of condensed tannins on the site of digestion. Plants containing low concentrations of condensed tannins have often resulted in superior body-weight gains and nitrogen retention by sheep. This study showed that condensed tannins selectively protected amino acids essential to sheep from degradation in the rumen, resulting in a $62 \%$ increase in apparent absorption, compared with control sheep.

Rumen ammonia and grain degradation. Estimates of optimal rumen ammonia concentration range from 14 to $194 \mathrm{mg} \mathrm{NH}_{3}-\mathrm{N} / \mathrm{l}$. This variation may be attributable to the nature of the fermentable substrate provided to the microbial population, since the minimal $\mathrm{NH}_{3}-\mathrm{N}$ concentration required to maximize the rumen degradation rate of barley dry matter exceeded that for maize.

Zinc deficiency and muscle protein turnover. In the rat, growth depression caused by a $\mathrm{Zn}$-deficient diet appears to be due to a reduction in both intake and efficiency of utilization of food. The latter defect reflects a reduced activation of muscle protein synthesis by feeding as well as increased protein degradation when food intake falls. 